\title{
BMJ Open Diagnostic performance of mid-upper arm circumference to identify overweight and obesity in children and adolescents: a protocol for a systematic review and meta-analysis
}

\author{
Binyam Girma Sisay (D , ${ }^{1}$ Hamid Yimam Hassen, ${ }^{2}$ Seifu Hagos Gebreyesus ${ }^{1}$
}

To cite: Sisay BG, Hassen HY, Gebreyesus SH. Diagnostic performance of mid-upper arm circumference to identify overweight and obesity in children and adolescents: a protocol for a systematic review and meta-analysis. BMJ Open 2021;11:e044624. doi:10.1136/ bmjopen-2020-044624

- Prepublication history and additional material for this paper is available online. To view these files, please visit the journal online (http://dx.doi.org/10. 1136/bmjopen-2020-044624)

Received 08 September 2020 Revised 28 February 2021 Accepted 16 March 2021

Check for updates

(c) Author(s) (or their employer(s)) 2021. Re-use permitted under CC BY-NC. No commercial re-use. See rights and permissions. Published by BMJ.

${ }^{1}$ Department of Nutrition and Dietetics, School of Public Health, Addis Ababa University, Addis Ababa, Ethiopia

${ }^{2}$ Department of Public Health, Mizan-Tepi University, Mizan, Ethiopia

Correspondence to Mr Binyam Girma Sisay; binyamgirma3@gmail.com

\section{ABSTRACT}

Introduction Mid-upper arm circumference (MUAC) has been suggested as an alternative screening tool to identify overweight and obesity in children and adolescents. Several studies have examined the diagnostic performance of MUAC to identify overweight and obesity in children and adolescents. However, the existing literature shows a considerable variability in measures of diagnostic performance and hence makes it difficult to direct clinical and public health practice. Therefore, this systematic review and meta-analysis aimed to synthesise evidence on the performance of MUAC to identify overweight and obesity in children and adolescents.

Methods and analysis A systematic search of databases including PubMed, EMBASE, SCOPUS, Cochrane Database of Systematic Reviews, Cochrane CENTRAL, Web of Science, CINAHL and PsycINFO will be conducted. The search will cover all studies until 1 April 2021. Grey literature will also be retrieved from Google Scholar. Titles and abstracts will be screened by two independent reviewers. The Quality Assessment of Diagnostic Accuracy Studies 2 tool will be used to assess the risk of bias and clinical applicability of each study. To assess possible publication bias, we will use Deeks' funnel plot. We will investigate the sources of heterogeneity by visual inspection of the paired forest plots and summary receiver operating characteristic plots. The pooled summary statistics for the area under the curve, sensitivities, specificities, likelihood ratios and diagnostic ORs with $95 \%$ $\mathrm{Cl}$ will be reported.

Ethics and dissemination The underlying study is based on published articles thus does not require ethical approval. The findings of the systematic review and metaanalysis will be published in a peer-reviewed journal and disseminated in different scientific conferences and seminars.

PROSPERO registration number CRD42020183148.

\section{INTRODUCTION}

Childhood and adolescent overweight and obesity is a significant public health challenge with adverse physical and psychological outcomes. ${ }^{1}$ From 1975 to 2016, the global burden of childhood and adolescent obesity
Strengths and limitations of this study

- To the best of the authors' knowledge, this will be the first systematic review and meta-analysis that will synthesise evidence on the performance of midupper arm circumference to identify overweight and obesity in children and adolescents.

- Detailed subgroup analysis and sensitivity analysis will be undertaken.

- The review will include studies conducted in all contexts not bounded by region or country.

- As the authors are only fluent in Amharic and English, a translation of some articles published in other languages might lead to language bias.

- There could be inconsistent quality in the reporting of diagnostic performance measures.

has increased from $0.7 \%$ to $5.6 \%$ in girls and from $0.9 \%$ to $7.8 \%$ in boys. If current trends continue, by the year 2022, child and adolescent obesity is predicted to exceed moderate and severe underweight. ${ }^{2}$ In developed countries, the prevalence of overweight and obesity was $23.8 \%$ for boys and $22.6 \%$ for girls; whereas in low and middle-income countries $12.9 \%$ of boys and $13.4 \%$ of girls were overweight and obese in 2013 . $^{3}$

Childhood and adolescent obesity has been linked to immediate risks of adverse health outcomes such as high blood pressure, type 2 diabetes, high cholesterol, fatty liver disease, sleep apnoea and cholelithiasis. ${ }^{4}$ Adverse health outcomes associated with adolescent overweight and obesity extend beyond the adolescence period. Those who were obese in their adolescence have a higher risk of morbidity from colorectal cancer, hypertension, type 2 diabetes, gout, abnormal kidney function, polycystic ovary syndrome, asthma and obstructive sleep apnoea in their adulthood. ${ }^{67}$ 
Timely identification is a vital step in mitigating these adverse consequences of adolescent overweight and obesity. ${ }^{8}$ Anthropometric measurements are a commonly used method to identify overweight and obesity due to their convenience, less expensive and strong positive correlation with per cent body fat. ${ }^{9}$ Body mass index (BMI) z-score is the widely used anthropometric measure for screening overweight and obesity in children and adolescents in both public health and clinical practice. ${ }^{10}$ According to the WHO, for children aged less than 59 months overweight is defined as BMI z-score $>+2 \mathrm{SD}$ whereas obesity is defined as BMI $z$-score $>+3 \mathrm{SD}^{11}$; for children and adolescents aged 5-19 years overweight is defined as BMI z-score $>+1 \mathrm{SD}$ whereas obesity is defined as BMI z-score $>+2 \mathrm{SD} .{ }^{12}$ There are several practical barriers related to BMI z-score particularly in resourcelimited settings where equipment and trained personnel are scarce; also measuring equipment is relatively expensive to buy, maintain and require regular calibration. Additionally, it is time consuming to measure weight and height, and interpret the value with a reference chart. Further measuring BMI z-score at community level requires carrying a height board and weighing scales which are cumbersome to health professionals. ${ }^{13} 14$ The major limitation of BMI is that it reflects both fat and fat-free components of body weight, ${ }^{15}$ which does not provide an indication on fat distribution of individuals. Waist circumference (WC) is the commonly used index to assess central adiposity. ${ }^{16-18}$ Despite its benefits, some individuals may be hesitant or reluctant to remove their clothing, unable to differentiate visceral from subcutaneous fat, differences in cut-offs for various ethnicities and being affected by postprandial abdominal distention. ${ }^{19}$ Recently, mid-upper arm circumference (MUAC) has been suggested as an alternative screening tool to identify overweight and obesity in children and adolescents. ${ }^{140-22}$ MUAC is not affected by postprandial abdominal distention, it does not require removing clothes and shoes, measuring tapes are relatively inexpensive and colour-coded MUAC tapes can be used by non-numerate fieldworkers. ${ }^{21}$ This makes MUAC practical, simple and less expensive, compared with BMI z-score and WC. Although several studies have examined the diagnostic performance of MUAC to identify overweight and obesity in children and adolescents, the lack of summarised evidence in measures of diagnostic performance makes it difficult to guide clinical and public health practice. This systematic review and meta-analysis aims to summarise the current evidence available on the performance of MUAC to identify overweight and obesity in children and adolescents.

\section{METHODS}

\section{Protocol registration and reporting}

The protocol of this systematic review and meta-analysis was registered at the International Prospective Register of Systematic Reviews (PROSPERO) (registration number
CRD42020183148). This review protocol is prepared following the Preferred Reporting Items for Systematic Review and Meta-Analysis Protocols 2015. ${ }^{23}$

\section{Information source}

Primarily, some electronic databases and PROSPERO were searched to check for similar published or ongoing systematic reviews to avoid duplication. A systematic search will be performed using the following electronic bibliographic databases to retrieve peer-reviewed articles: PubMed, EMBASE, SCOPUS, Cochrane Database of Systematic Reviews, Cochrane CENTRAL, Web of Science, CINAHL, PsycINFO. To retrieve grey literature systematic search will be performed using Google Scholar. Also, the reference lists of the retrieved studies will be explored manually to identify relevant articles. Database search will be done from 1 March 2021 to 1 April 2021.

\section{Search strategy}

The following keywords and Medical Subject Headings will be used in the electronic database search: MUAC, mid-upper arm circumference, BMI, body mass index, weight to height, dual-energy X-ray absorptiometry, DEXA, bioelectrical impedance, air displacement plethysmography, skinfold thickness, electric impedance, densitometry, hydrodensitometry, child, children, adolescence, adolescent, adolescents, teen, school-age children, preschool children will be used as a combination of free text and thesaurus terms to search for eligible articles. The detailed search strategy is provided in online supplemental appendix 1 .

\section{Criteria for considering studies for this review}

\section{Inclusion criteria}

Studies will be considered eligible and included if they fulfil the following criteria: (1) studies that assessed the diagnostic performance of MUAC as an index test to identify overweight/obesity; (2) compared with reference standard such as BMI z-score, weight to height, WC, skinfold thickness, dual-energy X-ray absorptiometry, air displacement plethysmography, bioelectrical impedance and hydrodensitometry; (3) should be done on children and adolescents aged 2-19 years; (4) should report at least one measure of diagnostic accuracy including sensitivity, specificity, predictive values, likelihood ratios (LR), area under the curve (AUC) or information that can be used to calculate this value; (5) studies should use observational study design, such as cross-sectional, cohort, case-control; (6) studies published in any language will be included. Studies published in any language other than English will be translated to English.

\section{Exclusion criteria}

Studies will be excluded from the review for any of the following reasons: (1) duplicate publication of the same study, (2) articles available only in abstract form, letters, reviews, commentaries, editorials and case series. 


\section{Data management}

Articles from all comprehensive searches of databases, grey literature and relevant articles retrieved from the reference list of obtained articles will be exported as EndNote files (including titles and abstracts), which will then be imported into EndNote as a single library. Duplicate articles from the searches will be verified and removed. The remaining articles will be imported into rayyan.QCRI.org, ${ }^{24}$ a web-based tool that facilitates screening and collaboration among researchers.

\section{Study selection}

Two of the authors (BGS and HYH) will independently review the titles and abstracts of all obtained articles. Where there is a disagreement between the two reviewers regarding study inclusion it will be resolved by discussion and consensus; if consensus cannot be reached a third author (SHG) will participate and make the final decision whether to include the article or not. In case of any key missing information in the articles, authors will be contacted through emails. All reasons for exclusion of articles will be noted and the review process will be presented using the Preferred Reporting Items for Systematic Reviews and Meta-Analyses flow chart.

\section{Patient and public involvement}

Patients or the public will not be directly involved in the design or planning of this study.

\section{Assessment of methodological quality}

The methodological quality of the included studies will be evaluated using the Quality Assessment of Diagnostic Accuracy Studies (QUADAS-2) tailored for this review. ${ }^{25}$ QUADAS-2 evaluates the risk of bias and applicability through the use of signalling questions corresponding to the four key domains covering patient selection, index test, reference standard and flow and timing. To reduce uncertain risks, we will request clarification from authors when relevant information is missing. Two authors (BGS and $\mathrm{HYH}$ ) will independently evaluate the selected studies. A disagreement between the reviewers on individual items will be resolved by a consensus.

\section{Data extraction}

We will pilot test the data extraction form. After refining the data collection form based on the pilot test, two authors (BGS and HYH) will independently extract all relevant data from included studies. Extracted data will be crosschecked and any discrepancy will be resolved by discussion and consensus. If consensus cannot be reached, the third author will be consulted and a decision will be made.

We will retrieve the following data from included studies using a standardised data extraction form specifically developed for this systematic review and meta-analysis. The following data will be retrieved: first author's name, year of publication, country or region, funding source, study design, total sample size, number of males and females, response rate, age of study participants, MUAC cut-off values used to define overweight/obesity, reference standard used to determine overweight and obesity, diagnostic criteria of overweight and obesity (reference standard), sensitivity, specificity, AUC, and LR+ and LR-. We will also extract available data on true positive, false positive, true negative and false negative for overweight and obesity to construct a $2 \times 2$ contingency table. In case of lacking key information, we will contact the primary authors through email for the missing data.

\section{Summary measures}

The outcomes of primary interest in our review will be the sensitivity, specificity and AUC of MUAC to identify overweight and/or obesity in children and adolescents. Additional analyses will be done using LR+ and LR-, diagnostic OR and Youden's index.

\section{Statistical analysis and data synthesis}

The extracted data will be exported to STATA/SE V.16 for further processing and analysis. We will carry out statistical analyses according to recommendations provided in the Cochrane Handbook for Systematic Reviews of Diagnostic Test Accuracy. ${ }^{26}$ We will summarise the diagnostic test accuracy by creating a $2 \times 2$ table for each study based on information retrieved directly from the papers. We will perform a graphical descriptive analysis of the included studies. We will report coupled forest plots (sensitivity and specificity separately, along with the 95\% CIs), and we will provide a graphical representation of studies in the receiver operating characteristic (ROC) curve (sensitivity against 1-specificity).

We will use a hierarchical summary receiver operating characteristic (HSROC) curve model to produce summary receiver operating characteristic (SROC) curves. ${ }^{27}$ Area under the summary receiver operating characteristic (AUC-SROC) curve values with corresponding $95 \%$ CI will be determined to describe the overall level of accuracy. The following guidelines have been suggested for interpretation of AUC-SROC: an area of $\leq 0.5$ considered to have no discriminatory power, $>0.5$ and $\leq 0.7$ to have low discriminatory power, $>0.7$ and $\leq 0.9$ to have good discriminatory power and 1 to be a perfect test. ${ }^{28}$ We will estimate the pooled sensitivity and specificity from the parameter estimates of the HSROC model. In addition, we will derive the summary values of LR+ and LR-.

\section{Investigations of heterogeneity}

We will investigate the sources of heterogeneity by visual inspection of the paired forest plots and SROC plots. A threshold effect could also be one of the important causes of heterogeneity between studies. Variations in the diagnostic accuracy across studies may be partly due to variations in cut-off points. Hence, we will use the Spearman correlation coefficient between the logit of sensitivity and the logit of 1-specificity to detect the threshold effect. ${ }^{29}$ If adequate data are 
available, we will investigate the influence of covariates such as study design, cut-off point, different reference standards, race, age and gender of participants. We will perform either using subgroup analysis or meta-regression models whenever it is appropriate. If it is appropriate to combine studies, we will assess the covariate effect using the log LR test for comparison of models with and without the covariate term. We will consider $p$ values $<0.05$ as statistical significance.

The different reference standards used to identify children and adolescents with overweight and obesity may introduce heterogeneity. Reference standards based on relatively equivalent diagnostic performance comparable accuracy, studies that used dualenergy X-ray absorptiometry, hydrodensitometry and air displacement plethysmography will be grouped together and the pooled estimates will be reported and compared with those studies that used lower accuracy measures as the reference standard method (bioelectrical impedance, skinfold thickness and BMI).$^{30-32}$

\section{Assessment of publication bias}

To assess possible publication bias, we will use Deeks' funnel plot, with Deeks' asymmetry test, where $\mathrm{p}<0.05$ will be considered as significant asymmetry. ${ }^{33}$

\section{Sensitivity analyses}

We plan to assess the effect of risk of bias of included studies on diagnostic accuracy by performing a sensitivity analysis by excluding studies classified as having high or unclear risk of bias in at least one of the domains of QUADAS-2.

\section{Grading the quality of evidence}

The quality of evidence will be evaluated using the Grading of Recommendations Assessment, Development and Evaluation working group methodology for diagnostic tests and strategies. ${ }^{34}$ The quality of evidence will be evaluated across the domains of risk of bias, consistency, directness, precision and publication bias. Quality of evidence will be classified as high, moderate, low or very low.

\section{Reporting this review}

The systematic review will be reported in accordance with the Preferred Reporting Items for Systematic Review and Meta-Analysis of Diagnostic Test Accuracy Studies (PRISMA-DTA) statement with a flow chart highlighting the article screening process. ${ }^{35}$ A completed PRISMA-DTA checklist, search strategies and quality appraisal tools will be included in the published version of the review as supplemental material.

\section{Potential protocol amendment}

In case of any updates to the protocol, the date and details of the amendment including rationale will be recorded and used to update the study protocol and PROSPERO registration record.

\section{DISCUSSION}

This systematic review and meta-analysis will provide up-todate evidence on the diagnostic performance of MUAC to identify overweight and obesity among children and adolescents. MUAC is commonly used to identify severe acute malnutrition among young children (6-59 months of age) in resource-limited settings. ${ }^{36}$ MUAC has also been used for numerous years to assess nutritional status in conditions, such as famines or refugee crises, where height and weight measurements are difficult. ${ }^{37}$ Several articles proposed that MUAC varies across sex and age. MUAC is positively correlated with the age of the children and adolescents suggesting MUAC increase with the age. MUAC mean value varies by sex; a study conducted in Chinese children shows that boys have higher MUAC value than girls. ${ }^{22}$ As far as we are aware, there is scarcity of evidence on the variation of MUAC according to ethnic groups. However, since body size and fat distribution vary according to ethnic group, this might also hold true for MUAC. ${ }^{38}$

Recently, several studies have suggested MUAC as a screening tool for overweight and obesity among children and adolescents. However, there is no universally agreed on cut-off point of MUAC to screen overweight and obesity among children and adolescents. ${ }^{1420-22}$ Also there is a lack of comprehensive evidence on the diagnostic performance of MUAC as a screening tool for overweight and obesity. Hence, the present systematic review will present summarised evidence on the ability of MUAC to identify overweight/ obesity. Knowing the diagnostic performance of MUAC will facilitate the use of MUAC as a screening tool in clinical and public health practice.

\section{ETHICS AND DISSEMINATION}

The underlying study is based on systematic reviews of published articles thus does not require ethical approval. The findings of the systematic review and meta-analysis will be disseminated in different scientific conferences and seminars and will be published in a peer-reviewed journal.

Contributors BGS conceived and designed the study, literature search and drafted the protocol. HYH participated in designing the study and critical revision of the manuscript. SHG took part in the critical revision of the manuscript. All authors read and approved the final manuscript.

Funding The authors have not declared a specific grant for this research from any funding agency in the public, commercial or not-for-profit sectors.

Competing interests None declared.

Patient consent for publication Not required.

Provenance and peer review Not commissioned; externally peer reviewed.

Supplemental material This content has been supplied by the author(s). It has not been vetted by BMJ Publishing Group Limited (BMJ) and may not have been peer-reviewed. Any opinions or recommendations discussed are solely those of the author(s) and are not endorsed by BMJ. BMJ disclaims all liability and responsibility arising from any reliance placed on the content. Where the content includes any translated material, BMJ does not warrant the accuracy and reliability of the translations (including but not limited to local regulations, clinical guidelines, terminology, drug names and drug dosages), and is not responsible for any error and/or omissions arising from translation and adaptation or otherwise. 
Open access This is an open access article distributed in accordance with the Creative Commons Attribution Non Commercial (CC BY-NC 4.0) license, which permits others to distribute, remix, adapt, build upon this work non-commercially, and license their derivative works on different terms, provided the original work is properly cited, appropriate credit is given, any changes made indicated, and the use is non-commercial. See: http://creativecommons.org/licenses/by-nc/4.0/.

ORCID iD

Binyam Girma Sisay http://orcid.org/0000-0002-9310-8170

\section{REFERENCES}

1 O'Donohue WT, Benuto LT, Tolle LW. Handbook of adolescent health psychology. Berlin/Heidelberg: Springer, 2013.

2 Abarca-Gómez L, Abdeen ZA, Hamid ZA, et al. Worldwide trends in body-mass index, underweight, overweight, and obesity from 1975 to 2016: a pooled analysis of 2416 population-based measurement studies in 128.9 million children, adolescents, and adults. The Lancet 2017;390:2627-42.

$3 \mathrm{Ng} \mathrm{M}$, Fleming T, Robinson M, et al. Global, regional, and national prevalence of overweight and obesity in children and adults during 1980-2013: a systematic analysis for the global burden of disease study 2013. Lancet 2014;384:766-81.

4 Daniels SR. The consequences of childhood overweight and obesity. Future Child 2006;16:47-67.

5 Cote AT, Harris KC, Panagiotopoulos C, et al. Childhood obesity and cardiovascular dysfunction. J Am Coll Cardiol 2013;62:1309-19.

6 Must A, Jacques PF, Dallal GE, et al. Long-Term morbidity and mortality of overweight adolescents. A follow-up of the Harvard growth study of 1922 to 1935 . N Engl J Med 1992;327:1350-5.

7 Inge TH, King WC, Jenkins TM, et al. The effect of obesity in adolescence on adult health status. Pediatrics 2013;132:1098-104.

8 Javed A, Jumean M, Murad MH, et al. Diagnostic performance of body mass index to identify obesity as defined by body adiposity in children and adolescents: a systematic review and meta-analysis. Pediatr Obes 2015;10:234-44.

9 Wells JCK, Fewtrell MS. Measuring body composition. Arch Dis Child 2006;91:612-7.

10 de Onis M, Onyango AW, Borghi E, et al. Development of a who growth reference for school-aged children and adolescents. Bull World Health Organ 2007;85:660-7.

11 Organization WH. WHO child growth standards: length/height-forage, weight-for-age, weight-for-length, weight-for-height and body mass index-for-age: methods and development. Geneva: World Health Organization, 2006.

12 WHO. Growth reference for 5-19 years, 2019. Available: https://www. who.int/growthref/who2007_bmi_for_age/en/

13 Neovius M, Linné Y, Barkeling B, et al. Discrepancies between classification systems of childhood obesity. Obes Rev 2004;5:105-14.

14 Craig E, Bland R, Ndirangu J, et al. Use of mid-upper arm circumference for determining overweight and overfatness in children and adolescents. Arch Dis Child 2014;99:763-6.

15 Sweeting HN. Measurement and definitions of obesity in childhood and adolescence: a field guide for the uninitiated. Nutr J 2007;6:32.

16 Ben-Noun L, Sohar E, Laor A. Neck circumference as a simple screening measure for identifying overweight and obese patients. Obes Res 2001;9:470-7.

17 Liu A, Hills AP, Hu X, et al. Waist circumference cut-off values for the prediction of cardiovascular risk factors clustering in Chinese school-aged children: a cross-sectional study. BMC Public Health 2010;10:82.
18 Jaiswal M, Bansal R, Agarwal A. Role of Mid-Upper arm circumference for determining overweight and obesity in children and adolescents. J Clin Diagn Res 2017;11:SC05-8.

19 Nafiu OO, Burke C, Lee J, et al. Neck circumference as a screening measure for identifying children with high body mass index. Pediatrics 2010;126:e306-10.

20 Talma H, van Dommelen P, Schweizer JJ, et al. Is mid-upper arm circumference in Dutch children useful in identifying obesity? Arch Dis Child 2019;104:159-65.

21 Chaput J-P, Katzmarzyk PT, Barnes JD, et al. Mid-upper arm circumference as a screening tool for identifying children with obesity: a 12-country study. Pediatr Obes 2017;12:439-45.

22 Lu Q, Wang R, Lou D-H, et al. Mid-upper-arm circumference and arm-to-height ratio in evaluation of overweight and obesity in Han children. Pediatr Neonatol 2014;55:14-19.

23 Shamseer L, Moher D, Clarke M, et al. Preferred reporting items for systematic review and meta-analysis protocols (PRISMA-P) 2015: elaboration and explanation. BMJ 2015;350:g7647.

24 Ouzzani M, Hammady H, Fedorowicz Z, et al. Rayyan-a web and mobile APP for systematic reviews. Syst Rev 2016;5:210.

25 Whiting PF, Rutjes AWS, Westwood ME, et al. QUADAS-2: a revised tool for the quality assessment of diagnostic accuracy studies. Ann Intern Med 2011;155:529-36.

26 Macaskill P, Gatsonis C, Deeks J, et al. Cochrane Handbook for systematic reviews of diagnostic test accuracy. version 090 . London: The Cochrane Collaboration, 2010: 83.

27 Rutter CM, Gatsonis CA. A hierarchical regression approach to meta-analysis of diagnostic test accuracy evaluations. Stat Med 2001;20:2865-84.

28 Swets JA. Measuring the accuracy of diagnostic systems. Science 1988;240:1285-93.

29 Moses LE, Shapiro D, Littenberg B. Combining independent studies of a diagnostic test into a summary ROC curve: dataanalytic approaches and some additional considerations. Stat Med 1993;12:1293-316

30 Warolin J, Kantor J, Whitaker LE, et al. Comparative assessment of change in fat mass using dual X-ray absorptiometry and airdisplacement plethysmography. Clin Obes 2012;2:66-72.

31 Minderico CS, Silva AM, Teixeira PJ, et al. Validity of air-displacement plethysmography in the assessment of body composition changes in a 16-month weight loss program. Nutr Metab 2006;3:32.

32 Frisard MI, Greenway FL, Delany JP. Comparison of methods to assess body composition changes during a period of weight loss. Obes Res 2005;13:845-54.

33 Deeks JJ, Macaskill P, Irwig L. The performance of tests of publication bias and other sample size effects in systematic reviews of diagnostic test accuracy was assessed. J Clin Epidemiol 2005:58:882-93.

34 Schünemann HJ, Schünemann AHJ, Oxman AD, Brozek J, et al. Grading quality of evidence and strength of recommendations for diagnostic tests and strategies. BMJ 2008;336:1106-10.

35 Mclnnes MDF, Moher D, Thombs BD, et al. Preferred reporting items for a systematic review and meta-analysis of diagnostic test accuracy studies: the PRISMA-DTA statement. JAMA 2018;319:388-96.

36 Myatt M, Khara T, Collins S. A review of methods to detect cases of severely malnourished children in the community for their admission into community-based therapeutic care programs. Food Nutr Bull 2006;27:S7-23.

37 James WP, Mascie-Taylor GC, Norgan NG, et al. The value of arm circumference measurements in assessing chronic energy deficiency in third World adults. Eur J Clin Nutr 1994;48:883-94.

38 Freedman DS, Wang J, Thornton JC, et al. Racial/ethnic differences in body fatness among children and adolescents. Obesity 2008; 16:1105-11. 